\title{
Laying the Foundation for Cystic Fibrosis Therapy: An Interview with Michael Boyle
}

\author{
Kevin Davies* \\ Executive Editor, Human Gene Therapy
}

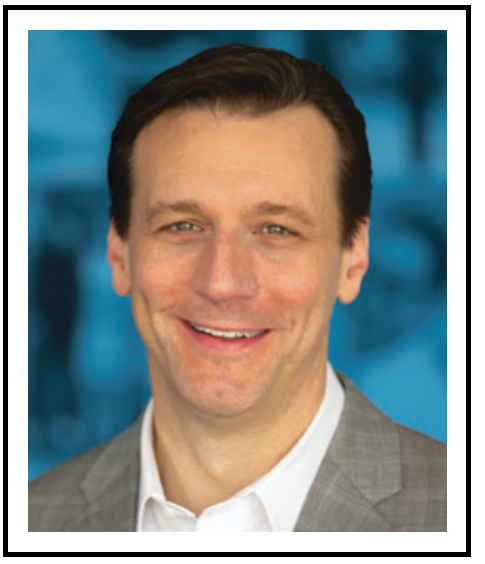

Michael Boyle, MD, President and CEO, Cystic Fibrosis Foundation
Editor's note: Few medical nonprofit organizations have had the sort of impact in developing genuine treatments as the Cystic Fibrosis Foundation (CF Foundation). Based in Bethesda, Maryland, the CF Foundation has championed cystic fibrosis (CF) researchers for decades, dating back well before the CF gene was identified in 1989. Since then, research has focused on better understanding the underlying pathophysiology of the genetic disease, and researching novel therapies, including gene therapies and small-molecule approaches. The Foundation's support has played a key role in the successful development of multiple CF drugs by Vertex Pharmaceuticals, which have significantly improved the life expectancy of many CF patients.

Physician-scientist Michael Boyle took over as president and CEO of the CF Foundation at the beginning of 2020. Here, in an exclusive interview with Human Gene Therapy Executive Editor Kevin Davies, Boyle looks back at the role of the Foundation, with particular emphasis on its support of gene therapy. Boyle also explains how interest in gene therapy and genome editing is growing, as the Foundation strives to leave no CF patient behind in its search for a cure.
Dr. Kevin Davies: Michael, please start by telling us a bit about your background and what you did before you came to the $\mathrm{CF}$ Foundation?

Dr. Michael Boyle: My background is as a physician-scientist. I started my medical training at Johns Hopkins as a medical student in 1986 and stayed at Hopkins for 29 years until I was a full professor in pulmonary and critical care medicine.

An interesting bit of background: as a pulmonary fellow, my research was in CF gene therapy. Much of my equipment had your Chief Editor Terry Flotte's name on it, including my ice bucket, which I used every day! So I saw Terry's name regu- larly, because he had been at Hopkins and left shortly before I arrived in that laboratory.

I started and directed the Johns Hopkins adult CF program for 15 years and was the principal investigator for several of the Vertex cystic fibrosis transmembrane regulator (CFTR) modulator trials. I assumed I would stay at Johns Hopkins for the rest of my career but had the opportunity to lead clinical research at the Foundation $\sim 5$ years ago- probably the one job that I was willing to leave for. I initially oversaw the CF Foundation's clinical research and therapeutic development network of 92 academic centers, and then started as president and CEO in January 2020. And what a year it has been!
Dr. Davies: Give us a snapshot of the CF Foundation. What do you spend on research over the course of the year?

Dr. Boyle: We have $\sim 700$ employees. We are based in Bethesda, Maryland, and we have local chapters supporting the $\mathrm{CF}$ community in almost every state in the United States. Our science really comes from two places - the Bethesda office, where we review the science, issue grants, and form collaborations with companies, and our laboratory in Boston, which has $\sim 45$ scientists. The laboratory focuses on assisting both companies and researchers in $\mathrm{CF}$, particularly in genetic technologies. Overall we

\footnotetext{
*Correspondence: Dr. Kevin Davies, Executive Editor, Human Gene Therapy, New Rochelle, NY 10801, USA. Email: kdavies@liebertpub.com
} 
spend $\sim \$ 200$ million each year on $\mathrm{CF}$ research.

Our goal is to advance therapies for people with $\mathrm{CF}$, to support the care of patients, and help address the other challenges that come with having CF. We also support a care center network and play a role in connecting and advocating for patients. But our primary goal is to advance the treatments and the cure of $\mathrm{CF}$.

Dr. Davies: What is the current state of play with the Vertex drugs that are now on the market and what proportion of $\mathrm{CF}$ patients are they able to treat? What difference have these drugs made in terms of the prognosis and lifespan of CF patients?

Dr. Boyle: It has been an amazing story! For the CF Foundation, a big focus early on was identifying the gene in 1989. All along, the intention was to use that information to develop therapies. As understanding advanced on the protein product of the gene, a chloride channel in lung epithelia, it was recognized that there was an opportunity to directly address the protein misfolding that occurred in the most common types of $\mathrm{CF}$.

There are $\sim 1,700$ different mutations that can cause $\mathrm{CF}$, and although those have a wide variety of effects on the protein, many cause misfolding. CFTR modulators-this new class of drug that has revolutionized the treatment of $\mathrm{CF}$-help to specifically address that misfolding. This started with work that the Foundation supported with Aurora Biosciences, identifying early leads for compounds that could refold the protein and open the channel. Vertex Pharmaceuticals took over that program and has done a remarkable job of rapidly advancing these transformative therapies.

Over the past decade, the percentage of patients able to be treated by CFTR modulators has steadily increased. Initially, it was a small percentage, maybe $5 \%$, with a drug called ivacaftor, which addressed rare gating mutations by increasing opening time of the channel. Over time, a two-drug combination of lumacaftor and ivacaftor allowed initial treatment of the most common folding mutation-F508del.

The big breakthrough however, $\sim 1$ year ago, was the approval of a three-drug combination, which dramatically restores CFTR function for anybody with $\mathrm{CF}$ who has a single F508del mutation. The early treatments for F508del required patients to be homozygous, because there was a gene dose effect.

This new three-drug combination of elexacaftor, tezacaftor, and ivacaftor called Trikafta, is strong enough to restore CFTR function to $\sim 50 \%$ of its wild-type levels, which is sufficient to make a dramatic clinical improvement in anybody with $\mathrm{CF}$ and a single F508del mutation. Approximately $90 \%$ of people with $\mathrm{CF}$ have this mutation and, therefore, have the potential to significantly benefit from this three-drug combination. That is why it has been such an amazing story!

The specific clinical benefits of this treatment include a large increase in lung function, return of their sweat chloride, which is the diagnostic test, into a nondiagnostic range, weight gain, and fewer problems with infection. Current median predicted survival is $\sim 45$ years and we fully expect that number will increase significantly in years to come.

We always say, however, that benefitting $90 \%$ of people with $\mathrm{CF}$ is not good enough. Despite CFTR modulators, many people with $\mathrm{CF}$ still suffer from challenging complications, and the role of the $\mathrm{CF}$ Foundation is to make sure we treat-and eventually cure-100\% of people with CF. That last $10 \%$ will require other approaches, because a significant portion of that group has mutations that do not make protein. This group is going to require genetic approaches-including approaches such as read-through agents of premature truncation codons (PTCs) to restore CFTR function, and RNA or DNA replacement.

Dr. Davies: These CFTR modulators are small molecules that work by helping coax CFTR to fold in the correct way. Who deserves the credit for devising this therapeutic paradigm?

Dr. Boyle: It has been a team approach. We talk about two types of modulators-the potentiators, which actually open up the channel to allow chloride transport-and correctors, which help with refolding of the protein to keep it from being targeted for degradation by the cell. The three-drug combination is a mixture of two correctors and a potentiator.

The credit belongs to a large group of scientists: those who discovered the gene, Francis Collins, Lap-Chee Tsui, and Jack Riordan. There was then a whole group of scientists who worked on understanding the protein, including people such as Mike Welch, Rick Boucher, and Bill Skach, our chief scientific officer. Foundation leaders including Bob Beall and Preston Campbell worked initially with Aurora Biosciences and later Vertex scientists Paul Negulescu and Fred Van Goor, who identified the smallmolecule potentiators and correctors. Finally, the CF Foundation's 92-academic center clinical trial network should be mentioned, as the investigators in the network performed all of the clinical trials.

\section{Dr. Davies: Let us talk about gene therapy. The CF Foundation was, I believe, quite heavily involved and there was a lot of excitement about gene therapy as an approach in the early 90s. What is your cur- rent thinking about gene therapy for CF?}

Dr. Boyle: I think you have identified something that has a key point. 
After the discovery of the CF gene, there was an initial wave of excitement that we are going to be able to quickly solve this. There was a somewhat infamous prediction that within 5 years we would likely see a cure for $\mathrm{CF}$ through gene therapy. Looking back, it taught us we have to be cautious about such statements because it is always more difficult and more complicated than you initially think. There were numerous gene therapy projects early on, including multiple clinical trials. Unfortunately, we experienced a little dose of reality.

Initial work with adenovirus and adeno-associated virus (AAV) all ran into obstacles-inflammation, limited levels, and duration of expression. People realized that it was not going to be as easy as we thought. There was a shift to other therapeutic approaches including CFTR modulators. Overall there have been $>20$ trials of gene therapy in $\mathrm{CF}$, all of which have showed perhaps modest expression but no clear clinical benefit and insufficient duration of effect. There has also been recognition that there are challenges that are specific for lung gene therapy and $\mathrm{CF}$, including cell turnover of lung epithelia and tenacious $\mathrm{CF}$ mucus.

The flip side, however, is that there has been continued clear progress in gene therapy in other diseases. These advances and proof of concept from other diseases, as well as the requirement in $\mathrm{CF}$ to develop genetic approaches to assure we can treat every person with $\mathrm{CF}$-no matter what their mutation-have led to a renewed focus on $\mathrm{CF}$ gene therapy.

This past November, at our North American $\mathrm{CF}$ conference, the $\mathrm{CF}$ Foundation simultaneously announced the data on the three-drug modulator combination, and the launch of our Path to a Cure initiative. This is a $\$ 500$-million program for the next 5 years focused on advancing genetic technologies, which will help us to develop therapies for
$100 \%$ of people, no matter what their mutation. Right at the heart of this is gene therapy.

Dr. Davies: Lets talk a bit more about the gene therapy and even the gene editing approaches. Now you are using the AAV vector. Is the plan simply to deliver the full protein? It is a big protein-how would that work?

Dr. Boyle: Let us talk about gene transfer first. We know there has been success with AAV in other organ systems including retina and muscle. There is an opportunity in $\mathrm{CF}$ if we are able to address some of the unique challenges that the lungs face.

AAV is our main approach currently. We recently made large awards to two AAV companies, Spirovant and 4D Molecular, for a total of up to $\$ 20$ million to advance CF AAV therapy. There are some challenges however. AAV allows a cargo capacity of $\sim 4.8 \mathrm{~kb}$. CFTR cDNA is $4.5 \mathrm{~kb}$. So when you add the other required regulatory elements, it is actually too big. Work has included use of a minigene approacha truncated functional DNA.

There has also been some work in nonviral approaches-lipid nanoparticles. There was a large $\mathrm{CF}$ gene therapy trial completed a couple of years ago in the United Kingdom utilizing lipid nanoparticles. There is also interest in lentivirus, as it may help address the duration of expression challenge.

Dr. Davies: What about gene editing? We are seeing some very exciting work clinically and gene editing fronts using CRISPR-Cas9 and maybe base editing coming into the portfolio as well. Are there other approaches using either of those technologies that could work for CF?

Dr. Boyle: We think that gene editing has the potential to be the best long-term solution in CF. There has already been successful editing of mutant CFTR in cell lines. The challenges are well known-first, the safety issues. Then we are going to have to deliver to stem cells. Right now, we are supporting work to identify those key progenitor cells in the airway, because that is going to be a requirement. Another challenge is going to be in the delivery of CRISPR-Cas9 or whatever platform we end up using. The components may be big and require using dual vectors with AAV. I think there is a lot of promise in gene editing, but it is going to take significant work.

There is one other potential challenge in $\mathrm{CF}$, and that is the variety of 1,700 different mutations. We are not going to be able to do this one mutation at a time. Options for editing to reach a large number of $\mathrm{CF}$ patients include inserting a super exon or targeting the most common mutations.

Dr. Davies: Last year, there was a rather protracted saga where Vertex was trying to reach an agreement with the British National Health Service, to bring these transformative medicines to British CF patients. Did the CF Foundation play any role in resolving that impasse? I am sure you are not involved in telling Vertex how to price their drugsyou want to see them be rewarded for the pioneering $R \& D$ that they have done. But at the same time to see a large pool of $\mathrm{CF}$ patients potentially denied or delayed access to the drugs that you have helped fund must be a conflicted feeling?

Dr. Boyle: We would love every person with $\mathrm{CF}$ to be able to benefit from these transformative drugs. And you are right, we do not have a say in the price of the drugs. We have always tried to say there is a middle ground, which will allow people to have access to these drugs while still 
providing incentive for companies bringing forward transformational science.

Our goal is to cure CF. As exciting as these modulator drugs are, there are additional drugs that are going to be even better. We want there to be an incentive to bring forward new drugs and novel gene therapy and gene editing that will treat everybody with $\mathrm{CF}$ and eventually lead to that cure.

Dr. Davies: Given you are now funding \$200 million a year, have a \$500-million Path to a Cure program in place, have you been able to help other societies who are working to find cures for other Mendelian diseases? They may not have your assets they may have a much smaller patient population, but have you established some best practices to help other nonprofits?

Dr. Boyle: We frequently meet with other nonprofits. A couple of things come to mind.

First, we know that some of the science that we are helping to support, particularly related to nonsense mutations including PTC read through therapy, has potential to benefit a large number of genetic diseases. There are thousands of diseases that are caused by nonsense mutations and may directly benefit from the science we are supporting. That is going to help not just people with $\mathrm{CF}$, but also genetic illnesses everywhere.

Second is more specific for other foundations. What we say is learn from our model. There are a couple of things in the model to highlight. One of these is supporting basic science to make sure that the understanding needed to drive new therapies is present. This harkens back to our work to allow the initial discovery of the CF gene.

Another key is developing a collaborative relationship with industry. We have an incredible history of such collaboration through venture philanthropy. We have taken our funding and used it to support the best science available in small biotech or drug companies. We have incentivized them to focus on $\mathrm{CF}$ with funding, with expertise, through the CF clinical trial network, and with information from our patient registry. When we meet with other foundations, we highlight these components.

Dr. Davies: Finally, you are really open to new ideas to continue to elevate the basic science and develop new gene therapy or gene editing approaches. How should people get in touch with you?

Dr. Boyle: We support any research that we think could end up making a difference for people with CF. That includes academic research through traditional request for applications (RFAs) and grant cycles wherein any researcher can apply. We recently had a Path to a Cure RFA specifically focused on supporting all aspects of genetic technologies, from basic research to delivery issues.
At the same time, we have an entirely separate program intended for industry called therapeutic development agreements (TDAs). This is for companies that have technology or therapeutic programs that may benefit people with CF. We do not have TDA deadlines. There is opportunity for millions of dollars of support, depending on the quality and stage of the research, and the potential for making a difference for people with CF.

Most recently, we have started a new incubator fund in collaboration with Longwood Fund, a biotechfocused venture capital firm, for companies with genetic technologies who are just being formed to incentivize them to focus on CF.

For the Path to the Cure program, we have a couple of key research areas that we are interested in. For gene therapy, we are particularly interested in expression and delivery issues. We need to have sufficient expression and efficiency of delivery to the airway epithelium. We need tropism for the tissues most affected by CF. And we need to address long-term expression issues, including immune response and neutralizing antibodies. For gene editing, we are very interested in identifying airway progenitor cells. This is going to be a requirement for successful lung gene editing in the future.

For all of these research programs, our website at www.cff.org is the place to start. Hope to see readers of $H G T$ there! 